\title{
Clinical significance of rapid ventricular tachycardia ( $>270$ beats per minute) provoked at programmed stimulation in patients without confirmed rapid ventricular arrhythmias
}

\begin{abstract}
Rapid uniform ventricular tachycardia (VT) ( $>270$ beats/min) or ventricular flutter induced during electrophysiological studies is thought not to be clinically significant in patients without cardiac arrest or documented rapid VT. The purpose of the study was to follow up 73 patients with inducible ventricular flutter but without confirmed rapid spontaneous VT. A long follow up (mean 3.5 years) identified two groups of patients. The first group had an excellent outcome and was characterised by a normal 24 hour Holter monitoring. In the second group, however, the risk of cardiac mortality was high (35\%) and spontaneous VT was $<270$ beats/min $(26 \%)$ and was characterised by couplets or salvos of extrasystoles on Holter monitoring. In this group the history of syncope and decreased left ejection fraction increased the risk of mortality and VT. The presence of late potentials increased the risk of spontaneous VT. Electrophysiologically guided antiarrhythmic therapy reduced the risk of VT.

Ventricular flutter was a non-specific finding in patients with normal Holter monitoring. In contrast, in patients with salvos of extrasystoles, ventricular flutter was associated with a high risk of cardiac mortality and VT.
\end{abstract}

(Br Heart f 1993;69:20-25)

Department of Cardiology, Brabois University Hospital, Vandoeuvre Les

Nancy, France

B Brembilla-Perrot

A Terrier de la Chaise

M Takoordial

C Suty-Selton

J Luc Brua

B Thiel

Department of

Epidemiology,

Brabois University

Hospital, Vandeouvre

Les Nancy, France.

$S$ Briançon

Correspondence to:

Dr Beatrice

Brembilla-Perrot,

Cardiologie, Chu De

Brabois, 54500 Vandoeuvre,

France.

Accepted for publication 7 August 1992
Ventricular fibrillation (VF) induced during electrophysiological studies is believed to be a non-clinical response in patients without clinical fibrillation ${ }^{12}$ whereas induced multiform ventricular tachycardia (VT) is an useful index of electrical instability in patients with cardiac arrest and haemodynamically symptomatic VT. ${ }^{3}$ The clinical significance of inducible rapid uniform VT or ventricular flutter in patients without a history of cardiac arrest or rapid VT is less established. Some reports claim that the induction of ventricular flutter after myocardial infarction is of no clinical significance, ${ }^{45}$ unlike the induction of a uniform and relatively slow arrhythmia. The significance of ventricular flutter when it is associated with another underlying heart disease is not known.

We analysed data from 73 consecutive patients who had inducible ventricular flutter and who did not have history of cardiac arrest or rapid VT and compared the results with their clinical outcome.

\section{Patients and methods}

PATIENTS

Ventricular flutter was induced by ventricular stimulation in 73 (mean (SD) age 57 (12), range 36-77) of the 1100 consecutive patients who had electrophysiological testing between January 1982 and December 1989 because of syncope, dilated cardiomyopathy, or myocardial infarction. For the purpose of this analysis, ventricular flutter was defined as having a cycle length of $<214 \mathrm{~ms}$, being uniform on a 12 lead electrocardiogram, having an uniform QRS configuration and axis, having constant rate, and requiring overdrive pacing or defibrillation because of circulatory collapse and loss of consciousness. All patients with spontaneous cardiac arrest or documented rapid VT were excluded.

Sixty patients had coronary disease and a history of myocardial infarction. Five had idiopathic dilated cardiomyopathy, three had hypertrophic cardiomyopathy, two had mitral valve prolapse, and one had corrected tetralogy of Fallot. Two patients had no clinica evidence of heart disease.

The indication for ventricular stimulation was unexplained syncope or dizziness (27 patients) and systematic evaluation at least one month after myocardial infarction to identify patients at risk of ventricular tachycardia $(n=41)$ or for systematic evaluation of patients with idiopathic dilated cardiomyopathy $(n=5)$.

\section{ELECTROPHYSIOLOGICAL STUDIES}

After they had given their informed consent patients were studied in a fasting non-sedated state. Antiarrhythmic therapy was discontinued for at least four half lives before the study. Three quadripolar or bipolar electrode catheters were inserted percutaneously and were positioned in the heart under fluoroscopic guidance. The right ventricle was stimulated at the apex and infundibulum with a programmable stimulater (Explorer 2000 Ela) that delivered square wave electrical impulses of $1.8 \mathrm{~ms}$ duration with the amplitude set at twice the diastolic threshold.

Our protocol for ventricular premature 
Table 1 Clinical characteristics and outcome in patients with inducible ventricular flutter

\begin{tabular}{lllll}
\hline Variable & $\begin{array}{l}\text { Cardiac } \\
\text { mortality } \\
(n=14)\end{array}$ & $\begin{array}{l}S D \\
(V T-V F \text { excluded }) \\
(n=6)\end{array}$ & $\begin{array}{l}V T \\
(+V F) \\
(n=10)\end{array}$ & $\begin{array}{l}\text { Alive } \\
(V T \text { excluded } \\
(n=53)\end{array}$ \\
\hline Age (yr) (mean (SD)) & $52(15)$ & $37(17)$ & $57(17)$ & $57(11)$ \\
Syncope (\%) & $10 / 14(71 \%)$ & $4 / 6(67 \%)$ & $7 / 10(70)$ & $14 / 53(26)$ \\
LVEF & $31(16)$ & $35(19)$ & $29(13)$ & $46(5)$ \\
Late potentials & $5 / 7$ & $1 / 2$ & $6 / 7$ & $15 / 35(43 \%)$ \\
Holter monitoring & $12 / 14(86 \%)$ & $6 / 6$ & $9 / 10$ & $15 / 53(32 \%)$ \\
$\quad$ (grade IVA-IVB) & & & & \\
Treatment follow up: & 3 & 1 & 4 & 39 \\
None & 3 & 2 & 0 & 7 \\
AA: protected & 3 & 3 & 6 & 7 \\
AA: not protected & 8 & & &
\end{tabular}

SD, sudden death; VT, ventricular tachycardia; VF, ventricular fibrillation; LVEF, left ventricular ejection fraction.

^Patient was not given an antiarrhythmic drug (AA). Protected, VT induction was supressed by AA. Not protected, patient received AA drug but the VT was still inducible.

stimulation has been described elsewhere. ${ }^{6}$ Ventricular overdrive pacing was performed at cycle lengths of $500-300 \mathrm{~ms}$. Ventricular programmed stimulation was then performed at three cycle lengths (sinus and 600 , and $400 \mathrm{~ms}$ ) with one and two extrastimuli. If VT, ventricular flutter, or VF was not initiated, the next ventricular site was then stimulated in a similar fashion until VT, ventricular flutter, or VF lasting $\geqslant 30$ seconds or circulatory collapse was initiated.

SIGNAL AVERAGED ELECTROCARDIOGRAPHY

A signal-averaged electrocardiogram was obtained on the same day as programmed stimulation in 45 patients without bundle branch block. Recordings were processed and analysed according to the methods described by $\mathrm{Simson}^{7}$ by electrocardiograph developed by the Dr Ing Osypka GmbH.

We measured the magnitude of the high frequency signal in the last $40 \mathrm{~ms}$ of the QRS complex (V40) and the total QRS duration. Late potentials were defined as a root mean square of the amplitude of the signal that was $\leqslant 20 \mu \mathrm{V}$ in the last $40 \mathrm{~ms}$ of the filtered QRS. QRS duration was regarded as abnormal when it was > $120 \mathrm{~ms}$. Signals were analysed without knowledge of the clinical status of the patient.

\section{OTHER INVESTIGATIONS}

Ambulatory electrocardiographic monitoring

Twenty four hour ambulatory electrocardiographic recordings were analysed manually. The total number of extrasystoles each hour and every $24 \mathrm{~h}$ was noted, as was the maximum Lown grade.

\section{Radionuclide ventriculography}

The resting left ventricular ejection fraction (LVEF) was measured during sinus rhythm by ${ }^{99 \mathrm{~m}} \mathrm{Tc}$ radionuclide ventriculography and computer generated time activity curves.

\section{FOLLOW UP}

During follow up, 36 patients in whom frequent extrasystoles of Lown grade IVA or IVB were recorded during ambulatory electrocardiographic monitoring were started on antiarrhythmic drugs. Oral therapy was started with a class I drug and ambulatory electrocardiographic monitoring and serial programmed stimulation were used to determine the appropriate drug. If the class I drug was ineffective, amiodarone was tested and then a combination of $\beta$ blocker and amiodarone. All 36 patients were treated with a combination of at least a class I drug and amiodarone. At electrophysiological testing protection was defined as the inability to provoke either a sustained VT or a non sustained VT (> 15 repetitive responses). ${ }^{8}$ In 13 patients an effective drug regimen was identified. When the VT was still inducible the patient was treated with the drug that resulted in the disappearance of ventricular extrasystoles on the Holter monitoring, increased the tachycardia cycle length, and was tolerated best. ${ }^{9}$

\section{STATISTICAL ANALYSIS}

Continuous values were expressed as the

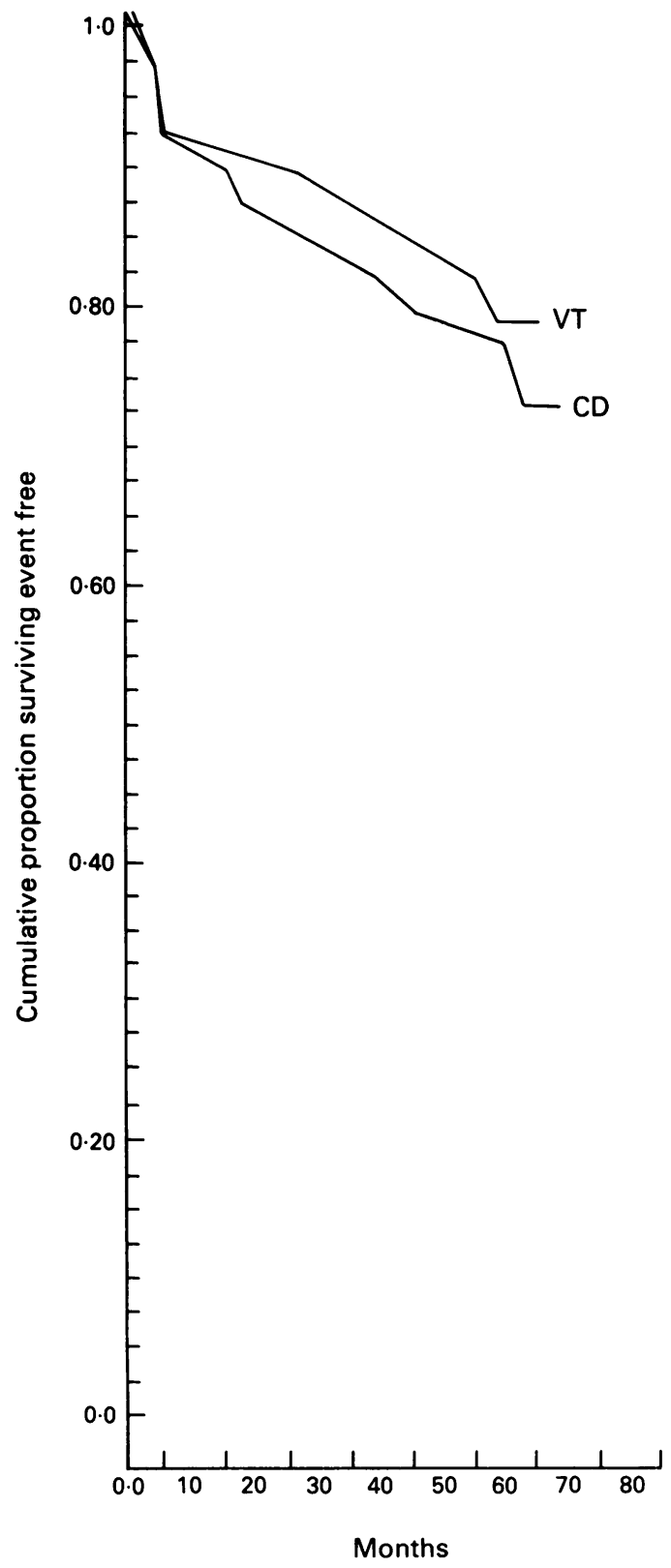

Figure 1 Life table analysis with survival curves for total cardiac deaths (CD) and for ventricular arrhythmic events (VT). 


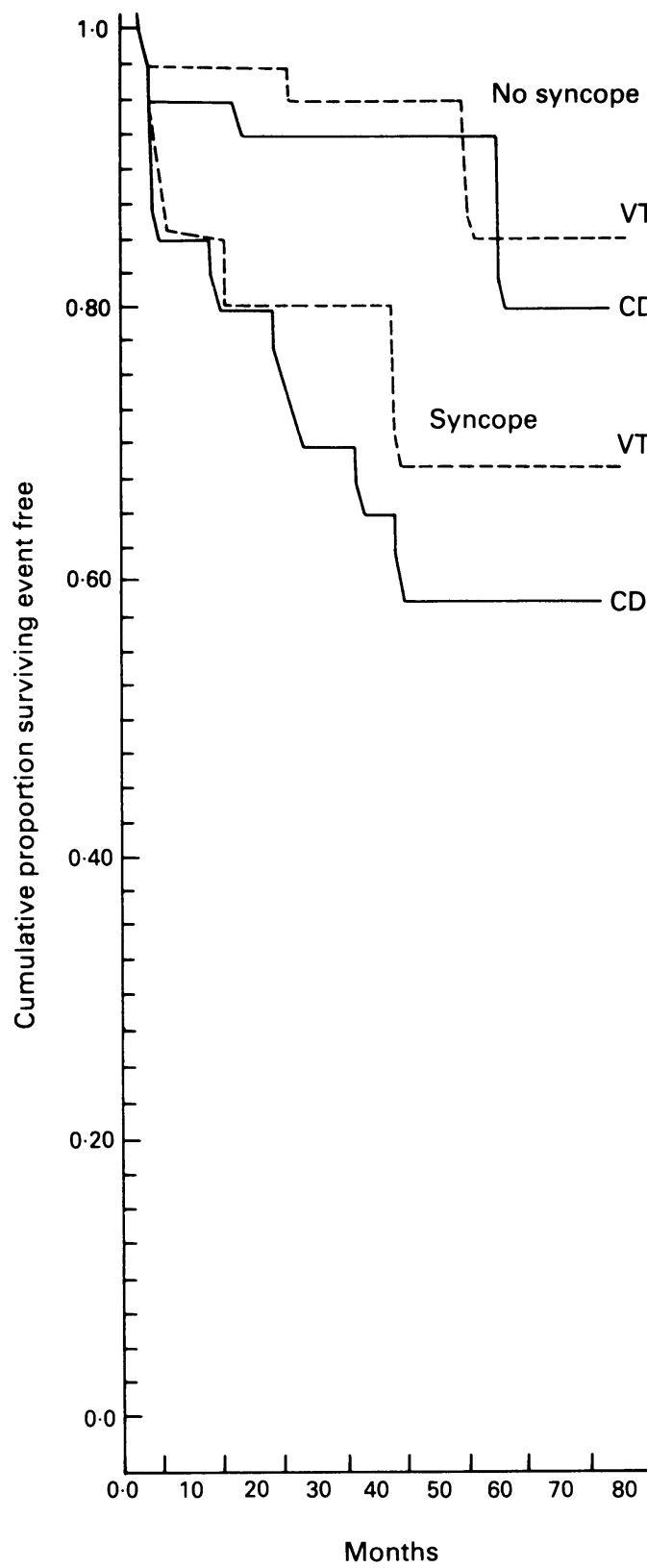

Figure 2 Life table analysis with survival curves for cardiac deaths and for ventricular arrhythmic events in patients with syncope and without. The Wilcoxon test showed significant differences in total cardiac survival $(p<0.01)$ and VT occurrence $(p<0.02)$ between those with syncope and those without.

mean (ISD). Continuous variables were compared by Student's two tailed $t$ test. Dichotomous variables were analysed by the $\chi^{2}$. We used stepwise logistic regression analysis to identify the independent variables predictive of VT and death. Survival curves were calculated by the Kaplan-Meier product limit method and compared by the generalised Wilcoxon (Breslow test). We used Cox's method to calculate the actuarial probabilities of survival, cardiac death, or VT. A $p$ value of $<0.05$ was considered significant.

\section{Results}

RESULTS OF INVESTIGATIONS

Ventricular premature stimulation-Seventy three patients had inducible sustained ventricular flutter. The mean duration of ven- tricular flutter before overdrive pacing or defibrillation was $18 \mathrm{~s}$. Sixty four patients required transthoracic direct current defibrillation with 200 to $400 j$ because of circulatory collapse and loss of consciousness. In the nine remaining patients ventricular flutter was $\underline{\underline{G}}$ stopped by overdrive pacing. The frequency $\frac{T}{\Phi}$ of ventricular flutter varied from 280 to 340 beats/min (mean 305 beats/min).

Signal-averaged electrocardiogram-Twenty seven patients had late potentials and the remaining patients $(n=23)$ had a normal signal-averaging.

Ambulatory electrocardiographic monitoringLown grade IVA or IVB ventricular arrhythmias were seen in 36 patients. VT was non sustained. All episodes of VT with $\geqslant 10$

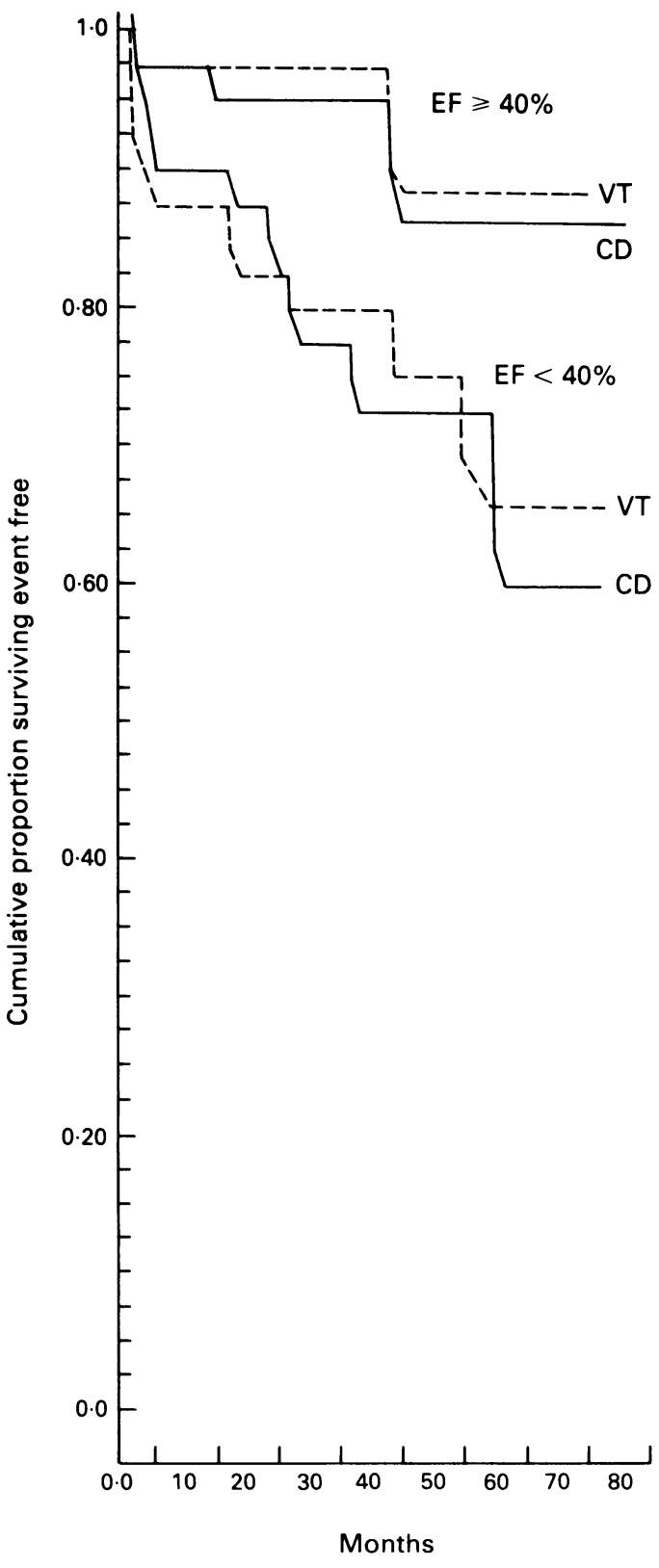

Figure 3 Life table analysis with survival curves for cardiac deaths and for ventricular arrhythmic events in patients with a left ventricular ejection fraction (EF) of $\geqslant 40 \%$ and $<40 \%$. There was no significant difference between these two groups in total cardiac survival $(p<0.07)$. Ventricular arrhythmic events $(V T)$ were $(p<0.07)$. Ventricular arrhythmic events $(V T)$ were
significantly more common in those with an $E F \geqslant 40 \%$ than in those with an EF of $<40 \%(p<0.02)$ 


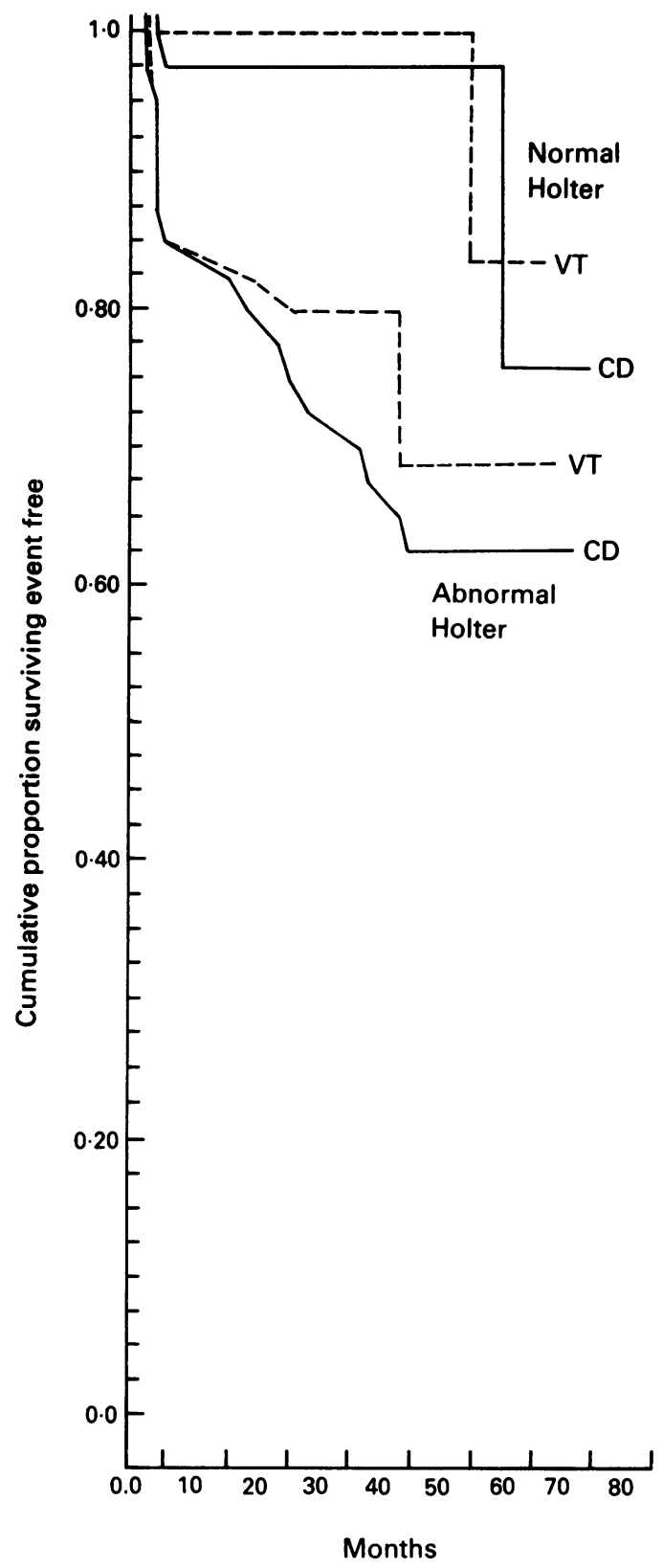

Figure 4 Life table analysis with survival curves for cardiac deaths and for ventricular arrhythmic events in patients with Lown grade IVA or IVB ventricular extrasystoles (abnormal Holter) and with Lown grade $O, I, I I$ or III activity (normal Holter). There were significant differences in total cardiac survival $(p<0.006)$ and VT occurrence $(p<0.004)$ between these two groups.

extrasystoles were excluded from this study.

Left ventricular ejection fraction-The mean left ventricular ejection fraction was $42(16 \%)$ (range 12 to $70 \%$ ). The ejection fraction was less than $40 \%$ in 31 patients.

\section{FOLLOW UP}

The mean follow up period was 3.5 years (up to 8 years) (table 1). During the follow up there were 13 deaths from cardiac causes (mortality 18\%). One death was related to cardiac transplantation. Nine were attributable to ventricular tachycardias either confirmed $(n=3)$ or presumed because of instantaneous death without prodromal symptoms $(n=6)$. Two patients died of heart failure. Another patient died of a non-cardiac cause.

In 10 patients spontaneous relatively slow VT ( $<250$ beats/min) developed, in three this was complicated by ventricular fibrillation and caused death. VT developed one month to five years after the induction of ventricular flutter. One of these patients underwent cardiac transplatation and died.

Curves to show survival without cardiac death and without arrhythmic events (confirmed ventricular tachycardia or ventricular fibrillation or both) were calculated by the Kaplan- Meier product limit method (fig 1). The actuarial probability of survival without cardiac death was 90 (3)\% at one year, $85(4) \%$ at two years, and $74(7) \%$ at five years and the probability of freedom from arrhythmic events was 90 (3)\% at one year and $88(4) \%$ at two years.

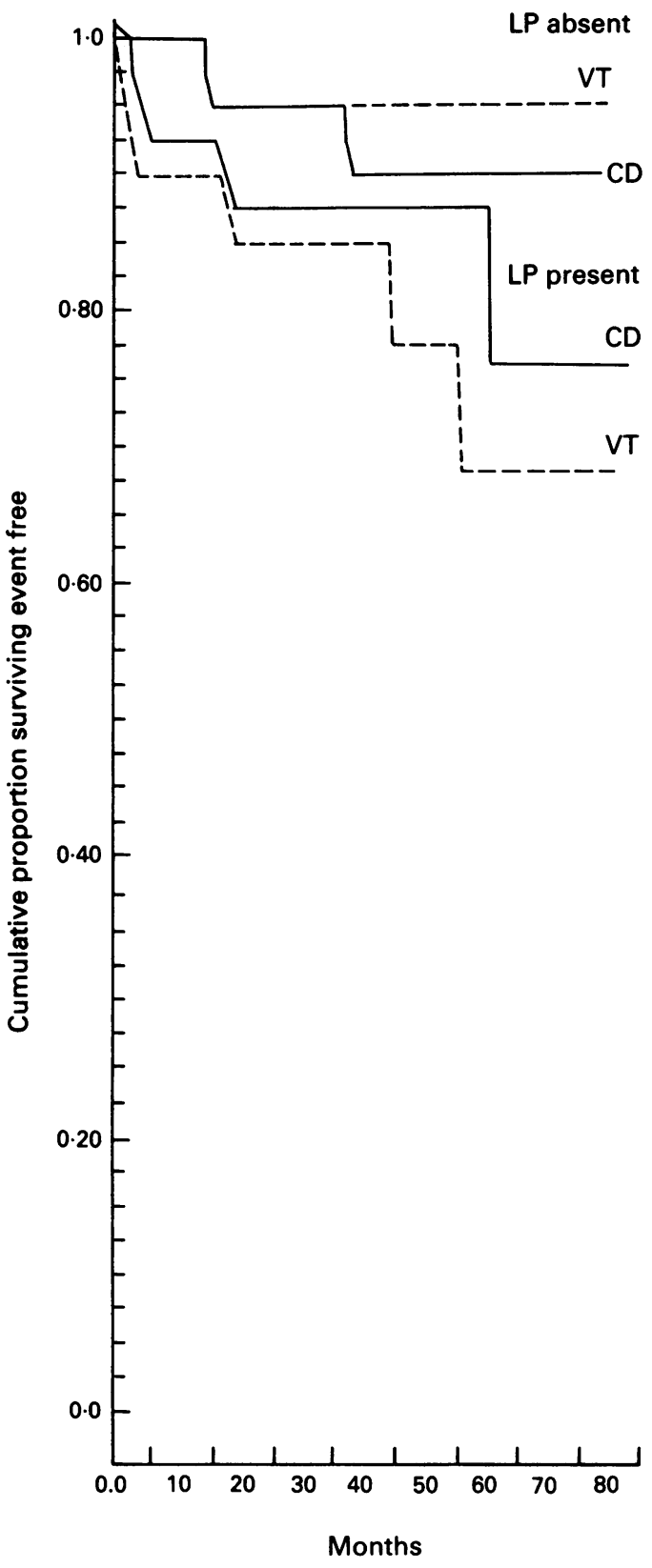

Figure 5 Life table analysis with survival curves for cardiac deaths and for ventricular arrhythmic events in patients with late potentials (LP) and without. There was no significant difference in total cardiac survival and VT occurrence between these two groups. 


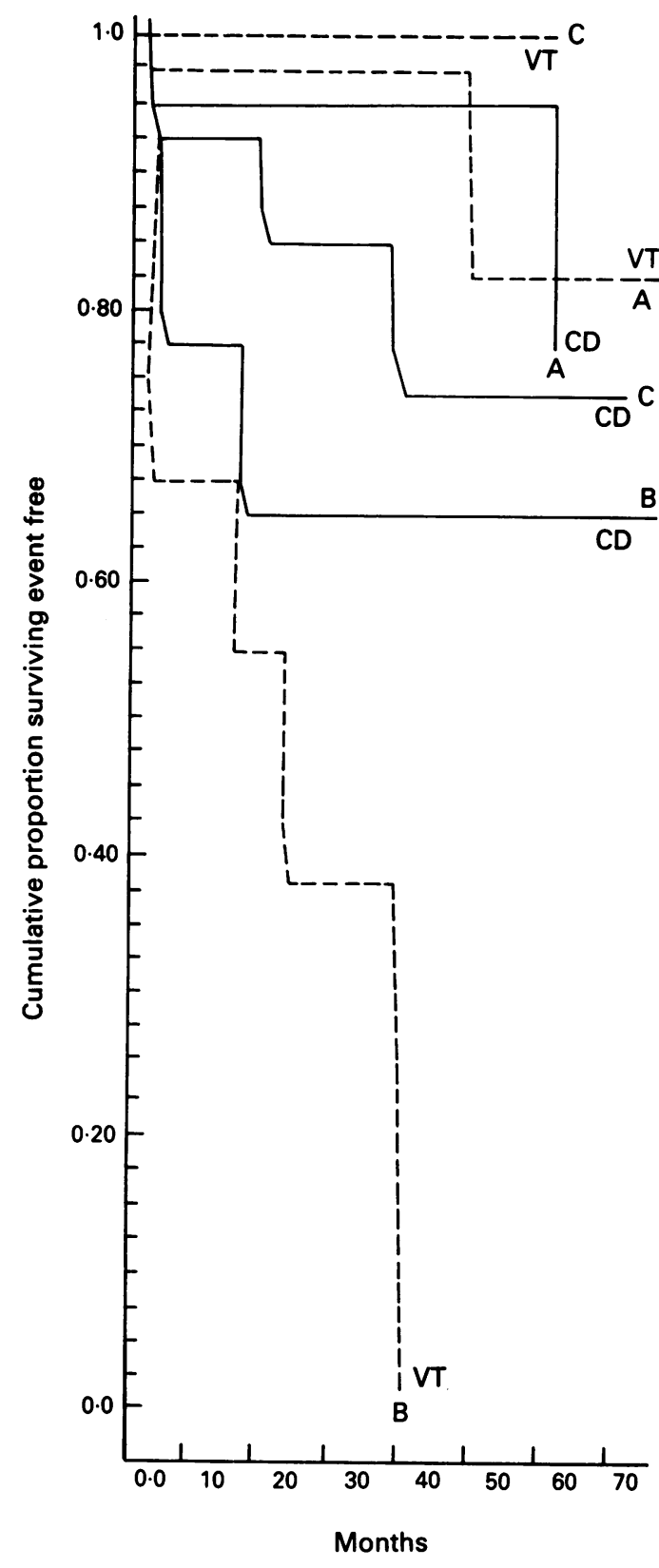

Figure 6 Life table analysis with survival curves for cardiac deaths and for ventricular arrhythmic events in patients treated with antiarrhythmic drugs $(A)$ treated with antiarrhythmic drugs and not protected, and treated with antiarrhythmic drugs and protected $(C)$. The risk of ventricular arrhythmic events was significantly reduced in group $C(p<0.001)$.

Patients with syncope had a significantly poorer prognosis $(p<0.01)$ and higher risk of ventricular arrhythmias $(p<0.02)$ than those without (fig 2). Patients with a left ventricular ejection fraction of $<40 \%$ had a poorer prognosis $(p<0.07)$ and significantly higher risk of ventricular arrhythmias $(p<0.02)$ than patients with an ejection fraction $\geqslant 40 \%$ (fig 3). According to Holter monitoring results, the group with Lown grade $4 \mathrm{~A}$ and $4 B$ ventricular ectopic activity have a significantly poorer prognosis $(\mathrm{p}<0.006)$ and a significantly higher risk of ventricular events $(p<0.004)$ than the group with Lown grades O, I, II, and III (fig 4). The presence of late potentials did not affect prognosis but the results did suggest a tendency to a higher risk of spontaneous VT $(p<0 \cdot 1)$ (fig 5). There may have been too few patients with late potentials for this difference to be statistically significant.
Table 2 Predictors of cardiac death (Cox regression analysis)

\begin{tabular}{llllll}
\hline & & \multicolumn{2}{c}{ Univariate } & & \multicolumn{2}{l}{ Multivariate } \\
\cline { 5 - 6 } Variables & No & $p$ Value & & $p$ Value & Coefficent $(S E)$ \\
\hline Syncope & 27 & $\mathrm{p}<0.01$ & & $\mathrm{p}<0.01$ & $132(0.7)$ \\
EF $\leqslant 40 \%$ & 31 & $\mathrm{p}<0.03$ & & $\mathrm{p}<0.09$ & $0.03(0.01)$ \\
Holter grade & & & & 0.03 \\
IV A-B & 36 & $\mathrm{p}<0.02$ & & 0.03 \\
\hline EF & &
\end{tabular}

$\mathrm{EF}$, left ventricular ejection fraction.

The prognosis and risk of ventricular arrhythmias were compared in patients who were not treated with antiarrhythmic agents (A), those who were treated but were not protected against VT (B), and those treated with antiarrhythmic agents who were pro- $\overrightarrow{0}$ tected (C) (fig 6). Group B had a poorer prognosis than groups $\mathrm{A}$ and $\mathrm{C}$ but the differences were not significant $(p<0.08)$. The risk of ventricular arrhythmic events was significantly $(p<0.001)$ reduced in group $C$. Cox regression multivariate analysis showed that only syncope $(p<0.01)$ and a low ejection fraction $(p<0.09)$ were independent variables predicting cardiac death in the follow up period (table 2). Cox regression multivariate analysis showed that only Lown grades IVA or IVB for ventricular ectopic activity $(p<0.005)$ were independent variables predicting ventricular arrhythmic events in the follow up period (table 3).

\section{Discussion}

The results of this study suggest that ventricular flutter initiated by programmed stimulation in patients without a confirmed history of rapid VT or VF had no clinical or prognostic significance when 24 hour monitoring did not show important ventricular arrhythmias and when patients did not have dizziness and syncope. In contrast, when these findings were associated with salvos of extrasystoles or with syncope mortality was increased.

The induction of sustained uniform VT $<270$ beats/min was reported to have a high diagnostic value in patients with spontaneous VT. ${ }^{10}{ }^{11}$ Two studies in symptom free survivors of acute myocardial infarction showed that inducible tachycardia predicted a significant risk of death or spontaneous tachycardia or fibrillation that was related to the cycle length of inducible VT. ${ }^{45}$ An event was more likely during the follow up if cycle length of the VT was $230 \mathrm{~ms}$ or more and the conclusions of both studies were that the induction of a ventricular flutter or fibrillation was of no clinical significance whereas the induction of

Table 3 Predictors of ventricular arrhythmic events (VT and VF) (Cox regression analysis)

\begin{tabular}{|c|c|c|c|c|}
\hline \multirow[b]{2}{*}{ Variables } & \multirow[b]{2}{*}{ No } & \multirow{2}{*}{$\frac{\text { Univariate }}{p \text { Value }}$} & \multicolumn{2}{|c|}{ Multivariate } \\
\hline & & & p Value & Coefficent (SE) \\
\hline $\begin{array}{l}\text { Syncope } \\
\text { EF } \leqslant 40 \% \\
\text { Holter grade }\end{array}$ & $\begin{array}{l}27 \\
31\end{array}$ & $\begin{array}{l}<0.15 \\
<0.01\end{array}$ & & \\
\hline $\begin{array}{l}\text { IV A-B } \\
\text { Sex F }\end{array}$ & $\begin{array}{r}36 \\
7\end{array}$ & $\begin{array}{l}<0.05 \\
<0.06\end{array}$ & $<0.005$ & $2.25(1.05)$ \\
\hline
\end{tabular}

$\mathrm{EF}$, left ventricular ejection fraction. 
a uniform and relatively slow arrhythmia was ( $<270$ beats $/ \mathrm{min}$ ).

We confirmed these results only in patients who did not have spontaneous ventricular arrhythmias on Holter monitoring. The prognosis was excellent in these patients. One patient, however, developed spontaneous VT, only five years after the induction of ventricular flutter. It is difficult to interpret the significance of this VT, because the substrate for the ventricular arrhythmias may have changed during this long follow up. This patient had abnormal late potentials at the time of the induction of ventricular flutter

In our study there was a high incidence of spontaneous relatively slow VT $\quad<270$ beats/min) in patients with inducible ventricular flutter and late potentials. The risk of spontaneous VT in patients with late potentials is well established ${ }^{412}$ but there are reports that late potentials are rare in patients with rapid spontaneous or inducible VT (>270 beats $/ \mathrm{min}) .{ }^{451314} \mathrm{We}$ found that late potentials were rarer $(43 \%)$ in survivors of acute myocardial infarction with normal Holter monitoring than in patients with inducible VT $<270$ beats $/ \mathrm{min}$ but its incidence in patients with spontaneous extrasystoles was high and these patients may be at risk of VT developing independently of the induction of ventricular flutter.

Our study showed that patients with syncope and inducible flutter had a bad prognosis. When syncope was not associated with spontaneous ventricular arrhythmias on Holter monitoring, however, only one patient died from non-arrhythmic cardiac death. The history of syncope was associated with an increased risk of cardiac death or VT only in patients with abnormal Holter monitoring. Also though some studies showed that electrophysiological study can identify patients at risk of sudden death and $\mathrm{VT}^{15-17}$ the results of our study suggest that the induction of a rapid VT ( $>270$ beats $/ \mathrm{min}$ ) in a patient with unexplained syncope and normal Holter monitoring has a good prognosis when left untreated.

In contrast our study suggests that ventricular flutter in patients with confirmed extrasystoles occurring in couplets or in salvos is of clinical significance and may be used to guide antiarrhythmic therapy. The higher mortality in the patient group treated with class I antiarrhythmic agents might have been attributable to the side effects of antiarrhythmic agents. ${ }^{18}$ Treatment, however, was always guided by Holter monitoring and repeated electrophysiological testing. When an arrhythmogenic effect of antiarrhythmic treatment was suspected, the treatment was changed.

Successful antiarrhythmic therapy guided by electrophysiological testing reduced the risk of spontaneous VT but did not the risk of sudden death. Also some patients with high risk of sudden death because they had syncope, salvos of extrasystoles, or decreased ejection fraction or both may require more intensive therapy when antiarrhythmic agents do not suppress the induction of ventricular flutter.
In conclusion, the clinical significance of inducible ventricular flutter depended principally on the results of 24 hour Holter monitoring. In patients without significant ventricular arrhythmia on Holter monitoring, ventricular flutter initiated by stimulation seemed to be a non-specific finding and did not mandate antiarrhythmic therapy. In contrast, in patients with salvos of extrasystoles ventricular flutter was associated with a high risk of cardiac mortality and VT and required electrophysiologically guided antiarrhythmic therapy.

1 Mahmud R, Denker S, Lehmann MH, Tchou P, Dongas $\mathrm{J}$, Akhtar M. Incidence and clinical significance of ventricular fibrillation induced with single and double ventricular fibrillation induced with single and double

2 Dicarlo L, Morady F, Schwartz AB, Shen EN, Baerman JM, Kral RB, Scheinman MM, Sung RJ. Clinical significance of ventricular fibrillation-flutter induced by ventricular programmed stimulation. Am Heart $\mathcal{J}$ 1985;109:959-69.

3 Torres V, Flowers D, Somberg J. The clinical significance of polymorphic ventricular tachycardia provoked at elec trophysiological testing. Am Heart $\mathcal{F}$ 1985;110:17-23.

4 Breithardt G, Borggrefe M, Haerten K. Ventricular late potentials and inducible ventricular tachyarrhythmias as a marker for ventricular tachycardia after myocardial infarction. Eur Heart $\mathcal{f}$ 1986; 7(suppl A): 127-34.

5 Denniss R, Richards DA, Cody DV, Russell PA, Young AA, Cooper MJ, Ross DL, Uther JB. Prognostic significance of ventricular tachycardia and fibrillation induced at programmed stimulation and delayed potentials detected on the signal averaged electrocardiograms of detected on the signal averaged electrocardiograms of
survivors of acute myocardial infarction. Circulation survivors of acute

6 Brembilla-Perrot B, Terrier de la Chaise A, Isaaz $\mathrm{K}$, Maron F, Cherrier F, Pernot C. Inducible multiform ventricular tachycardia in Wolff-Parkinson-White syndrome. Br Heart $\mathcal{f}$ 1987; 58:89-95.

7 Simson MB. Use of signals in the terminal QRS complex to identify patients with ventricular tachycardia after myocardial infarction. Circulation 1981;64:235-42.

8 Kim SG, Felder SD, Figura I, Johnston DR, Mercando $A D$, Fisher JD. Prognostic value of the changes in the mode of ventricular tachycardia induction during therapy with amiodarone or amiodarone and a class Ia py with amiodarone or amiodarone and a class

9 Waller TJ, Kay HR, Spielman SR, Kutalek SP, Greenspan AM, Horowitz LN. Reduction in sudden death and total mortality by antiarrhythmic therapy death and total mortality by antiarrhythmic therapy
evaluated by electrophysiological testing; criteria of evaluated by electrophysiological testing; criteria of arrhythmia. $\mathcal{F}$ Am Coll Cardiol 1987;10:83-9.

0 Vandepol CJ, Farshidi A, Spielman SR, Greenspan AM Horowitz LN, Josephson ME. Incidence and clinical significance of induced ventricular tachycardia. $A m$ Cardiol 1980;45:725-31.

11 Livelli FD, Bigger J Th, Reiffel JA, Gang ES, Patton JN, Noethling PM, Rolnitzky LM, Gliklich JI. Response to programmed ventricular stimulation : sensitivity, specificity and relation to heart disease. Am $\mathcal{f}$ Cardiol 1982;50:452-8.

12 Nalos PC, Gang ES, Mandel WJ, Lodenheim ML, Lass Y, Peter TH. The signal-averaged electrocardiogram as a screening test for inducibility of sustained ventricular a screening test for inducibility of sustained ventricular tachycardia in high risk patients:

13 Freedman RA, Gillis AM, Keren A, Soderhom-Difatte V, Mason JN. Signal-averaged electrocardiographic late potentials in patients with ventricular fibrillation or ventricular tachycardia. Correlation with clinical arrhythmia and electrophysiological study. $A m \mathcal{F}$ Cardiol 1985;55:1350-63.

14 Denniss AR, Ross DL, Richards DA, Halley LK, Cooper MJ, Johnson DC, Uther JB. Difference between patients with ventricular tachycardia and ventricular fibrillation as assessed by signal-averaged ectrocardiogram, as assessed by signal-averaged electrocardiogram, radionuclide ventriculography and

15 Morady F, Higgins J, Peters RW, Schwartz AB, Shen EN, Bhandari A, Scheinman MM, Sauv MJ. ElectroBhandari A, Scheinman MM, Sauv MJ. Electrophysiologic testing in bundle branch block and

16 Doherty JA, Pembrook-Rogers D, Grogan W, Falcone

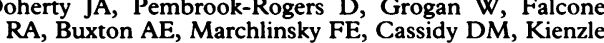
MG, Almendral JM, Josephson ME. Electrophysiologic evaluation and follow up characteristics of patients with recurrent unexplained syncope and presyncope. $A m$ Cardiol 1985;55:703-8.

17 Olshansky B, Mazuz M, Martins JB. Significance of inducible tachycardia in patients with syncope of unknown origin: a long term follow up. $\mathcal{F} \mathrm{Am}$ Coll Cardiol 1985;5:216-23.

18 The Cardiac Arrhythmic Suppression Trial (CAST). Investigators Preliminary Report : effect of encainide and flecainide on mortality in a randomised trial of Engl ₹ Med 1989;321:406-12. 